\title{
Formação de coalizão de defesa e atores chaves da política
}

\author{
Diana Cruz Rodrigues 1 \\ Mário Vasconcellos Sobrinho 12 \\ Ana Maria de Albuquerque Vasconcellos 1 \\ 1 Universidade da Amazônia / Programa de Pós-Graduação em Administração, Belém / PA - Brasil \\ 2 Universidade Federal do Pará / Programa de Pós-Graduação em Gestão de Recursos Naturais e Desenvolvimento Local, Belém / \\ PA - Brasil
}

\begin{abstract}
O artigo discute a formação de coalizão de defesa e o papel de atores chaves em políticas de Ciência, Tecnologia e Inovação (CT\&I) para inclusão social em um contexto subnacional. No âmbito do Modelo de Coalizão de Defesa (MCD), utilizou-se as categorias analíticas do subsistema da política e da coalizão de defesa como referência e buscou-se compreender as influências de atores chaves nestas, especificamente o policy broker (mediador) e o empreendedor de política. O subsistema de política foi delimitado pelo método de pesquisa baseada em caso e a análise de discurso foi mobilizada para compreender as crenças dos atores da política. A análise de dois casos do processo de política de CT\&I para inclusão social (tecnologia social e tecnologia assistiva) realçou os papéis de atores chaves na emergência dos subsistemas de política, porém estes tiveram diferentes efeitos sobre a formação de coalizões de defesa. O empreendedor da política teve maior relação com a formação de coalizão de defesa ao estabelecer mecanismos regulares para compartilhamento de crenças e aprendizados orientados para a política, bem como ao tomar iniciativas de coordenação da ação coletiva na coalizão de defesa nascente. O policy broker não alcançou relação positiva substancial com a formação das coalizões de defesa analisadas, embora tenha sido relevante para o agenda-setting e a permanência da pauta de inclusão social no processo da política. $\mathrm{O}$ artigo corrobora com a possibilidade de incorporação do conceito de empreendedor de política em análises do MDC e aponta características deste ator chave iluminadas pelo modelo analítico.
\end{abstract}

Palavras-chave: processo de políticas públicas; modelo de coalizão de defesa; política pública de ciência e tecnologia para inclusão social; tecnologia social; tecnologia assistiva.

\section{Formación de coaliciones de defensa y actores clave dentro de la política}

El artículo analiza la formación de coaliciones de defensa y los roles de los actores clave en las políticas de ciencia, tecnología e innovación (PCTI) para la inclusión social en un contexto subnacional. Considerando el modelo de coalición de defensa (MCD), se utilizaron como referencia las categorías analíticas del subsistema de la política y de la coalición de defensa, para comprender las influencias de los actores clave, específicamente del policy broker (intermediario) y del emprendedor de políticas. El subsistema de la política se delimitó mediante el método de investigación basado en casos y se usó el análisis del discurso para comprender las creencias de los actores en la política. El análisis de dos casos del proceso de la PCTI para la inclusión social (tecnología social y tecnología de la rehabilitación) resaltó los roles de los actores clave en la aparición de subsistemas de la política, sin embargo tuvieron diferentes efectos en la formación de coaliciones de defensa. El emprendedor de políticas tuvo una mayor relación con la formación de una coalición de defensa al establecer mecanismos regulares para comunicar sus posiciones y aprendizajes orientados a la política, así como al tomar iniciativas para coordinar la acción colectiva en la naciente coalición de defensa. El policy broker no logró una relación positiva con la formación de las coaliciones de defensa analizadas, pero sí fue relevante para el establecimiento de la agenda y la permanencia de la pauta de inclusión social en el proceso de la política. Este trabajo corrobora la posibilidad de incorporar el concepto de emprendedor de política en los análisis de MDC y señala las características de este actor clave en el modelo analítico estudiado.

Palabras clave: proceso de políticas públicas; modelo de coalición de defensa; política pública de ciencia y tecnología para la inclusión social; tecnología social; tecnología de la rehabilitación. 


\section{Advocacy coalition formation and key actors within the policy}

The article discusses advocacy coalition formation and the roles of key actors in science, technology and innovation (ST\&I) policies for social inclusion in a subnational context. The policy subsystem category and concept of advocacy coalition are used in the context of the advocacy coalition framework and address the need to understand the influences of key actors (policy broker and policy entrepreneur) on it. The policy subsystem was outlined using case-oriented research and the discourse was analyzed in order to understand the policy actors' beliefs. The analysis of two cases of ST\&I policy processes for social inclusion (assistive technology and social technology) highlighted policy broker and policy entrepreneur key roles in the emergence of policy subsystems, but had different effects on advocacy coalition formation. The policy entrepreneur had a closer relationship with advocacy coalition building when setting up regular mechanisms to share beliefs and policy-oriented learning, as well as taking initiatives to coordinate the collective action of members in the early advocacy coalition. Although relevant in agenda setting and maintaining a specific social inclusion agenda in the policy process, the policy broker did not achieve a positive relationship with advocacy coalition building. The article corroborates the possibility of incorporating the concept of policy entrepreneur in analyses of the advocacy coalition framework and highlights this actor's characteristics through this analytical model.

Keywords: policy process; advocacy coalition framework; public policy of science and technology for social inclusion; social technology; assistive technology.

\section{INTRODUÇÃO}

O artigo discute a formação de coalizão de defesa e o papel de atores chaves na ascensão de temáticas marginais no processo de política pública, a partir do modelo de coalizão de defesa (MCD) (JenkinsSmith, Nohrstedt, Weible, \& Ingold, 2017; Sabatier \& Jenkins-Smith, 1993; Weible, Sabatier, \& McQueen, 2009). Examina-se especificamente os processos de política de ciência, tecnologia e inovação (CT\&I) em contexto subnacional, particularmente os processos referentes ao fomento à tecnologia social (TS) e à tecnologia assistiva (TA) no governo estadual do Pará, região norte do Brasil.

No Brasil, pautas relacionadas a ciência e tecnologia (C\&T) para inclusão social ascenderam na agenda da política de CT\&I federal no início da década de 2000, principalmente ancoradas, à época, na Secretaria de C\&T para Inclusão Social (SECIS) (Dias, 2012). Embora essas pautas mantivessem uma posição marginal na agenda de CT\&I, iniciativas em diversas temáticas foram desenvolvidas, como em tecnologias assistivas e tecnologias sociais.

Tecnologia Assistiva (TA) refere-se ao desenvolvimento de recursos para pessoas com deficiência ou com mobilidade reduzida, os quais visam manter ou melhorar a autonomia e promoção de bem-estar dessas pessoas (MacLachlan et al., 2018). Tecnologia Social (TS) corresponde a produtos, técnicas ou metodologias desenvolvidas com a participação de comunidades locais que visam a transformação social (RTS, 2010). Frequentemente, TS está relacionada com o atendimento a necessidades básicas e melhoria de qualidade de grupos sociais em vulnerabilidade.

O estado do Pará encontra-se em um contexto periférico da política de CT\&I do Brasil, com baixos níveis de investimentos. A região norte onde o estado se localiza também apresenta, de forma geral, os mais baixos indicadores de CT\&I no país (Cavalcante, 2011; Theis, Strelon, \& Lasta, 2017). Estas condições são limitantes para temáticas marginais, como estas de C\&T para inclusão social, ascenderem à agenda.

Na agenda de CT\&I do estado Pará, as pautas de políticas de TA e TS emergiram somente a partir de 2011. A ascensão relativamente recente das pautas à agenda estadual, com considerável defasagem 
frente ao surgimento na agenda nacional, nos conduziu a examinar com maior cuidado os elementos internos do processo de política subnacional que foram decisivos para a mudança e o alcance destas pautas à agenda, apesar da existência de condições limitantes do contexto periférico, e as mantido no processo de política, ainda que marginalmente.

Neste cenário, buscamos analisar a trajetória das pautas de TA e TS no contexto da CT\&I subnacional do Pará, de 2011 até 2018, a partir do MCD, com foco na formação de coalizões de defesa locais e em atores chaves da política.

Entre os atores chaves, selecionamos o mediador da política (policy broker), o qual já era previsto no MCD desde a publicação seminal de Sabatier \& Jenkins-Smith (1993), e o empreendedor de política. A pertinência da análise de um conjunto mais diverso de atores chaves no estudo de coalizões de defesa, incluindo o empreendedor de política, foi realçado recentemente em Weible, Ingold, Nohrstedt, Henry e Jenkins-Smith (2019). Análises do papel do empreendedor da política em coalizões de defesa foram discutidas inicialmente em Mintrom e Vergari (1996) e em algumas publicações posteriores como Mintrom e Norman (2009) e Belánd e Cox (2015).

Embora o papel do empreendedor de política ainda não tenha sido incorporado em hipóteses nas revisões mais amplas do MCD (Weible et al., 2009; Jenkins-Smith, Nohrstedt, Weible, \& Sabatier, 2014; Jenkins-Smith et al., 2017), as publicações citadas demonstram que sua análise pode ser promissora em conjunto com o MCD, particularmente ao iluminar processos de construção das coalizões de defesa e de mudanças rápidas no processo da política.

Portanto, o artigo busca discutir a formação de coalizões de defesa e o papel de atores chaves (policy broker e empreendedor da política) nestas, enquanto elementos promotores de mudanças no processo de política. Especificamente, esta discussão se baseia na ascensão de pautas de inclusão social à agenda da política de CT\&I e movimentos de resistência dos atores da política para permanecer nesta agenda, mesmo que marginalmente, dando assim efetividade à mudança no processo da política ao longo do tempo.

Em termos aplicados, entendemos que o artigo também contribui para a ampliação de estudos de políticas de CT\&I em contextos subnacionais no Brasil, particularmente aqueles periféricos. O que favorece a geração de conhecimentos para mudanças em direções de elaborações de políticas de CT\&I mais endógenas aos âmbitos subnacionais, bem como de uma coordenação federativa mais sensível às especificidades e assimetrias regionais.

\section{MODELO DE COALIZÃO DE DEFESA}

O modelo de coalizão de defesa (MCD) surgiu na década de 1980 por trabalhos pioneiros de Paul Sabatier e Hank Jenkins-Smith (Sabatier \& Jenkins-Smith, 1993). O modelo pertence a um enfoque cognitivo sobre o processo de política, pela qual a racionalidade dos atores é direcionada para variáveis relacionadas às ideias, crenças e conhecimento (Faria, 2003; Jenkins-Smith et al., 2017).

O MCD enfatiza uma visão integral do processo da política, em que a politics é permeada de disputas de crenças e ideias dos atores sobre a definição dos problemas públicos, suas causas, soluções alternativas e formas de monitoramento e avaliação. As ideias e crenças vencedoras dessas disputas conformam a policy, refletindo-se em seus objetivos, desenhos preferenciais e no conteúdo de um domínio de política. 
Este artigo centra-se na categoria de subsistema da política, com foco no conceito de coalizão de defesa e no papel de atores em posições chaves no processo da política. O subsistema da política é a unidade de análise principal do processo de política no MCD, onde os atores da política atuam e possivelmente se organizam em coalizões de defesa, mediante suas crenças e recursos. Estes atores definem estratégias para influenciar as decisões governamentais (regras institucionais, alocações de recursos, nomeações), bem como os produtos e resultados da política pública (Jenkins-Smith et al. 2014).

O subsistema de política é concebido como um conjunto de atores envolvidos em um problema de política que interagem sistematicamente para influenciar o conteúdo e as direções desta política (Sabatier \& Jenkins-Smith, 1993). O escopo do subsistema é delimitado pelo mapeamento dos atores (e suas possíveis organizações) que buscam influenciar um determinado processo de política.

Cabe ressaltar que os atores da política no MCD não estão restritos ao chamado "triângulo de ferro" (legisladores, agentes do executivo e grupos de interesses) (Weible et al., 2019). A visão sobre os potenciais atores se expande a todos aqueles que buscam influenciar o processo da política, incluindo perfis como pesquisadores, consultores, jornalistas e agentes do judiciário (Capella \& Brasil, 2015).

As coalizões de defesa, por sua vez, são conceituadas como um conjunto de atores que compartilham crenças essenciais no âmbito da política e coordenam suas ações de uma forma não trivial, com o objetivo de influenciar o subsistema da política (Sabatier \& Jenkins-Smith, 1993; Jenkins-Smith et al., 2017).

O agrupamento de atores da política em coalizões de defesa envolve duas características principais de seu conceito original: compartilhamento de crenças e coordenação de ações. Destas características, o sistema de crenças se mantém como elemento teórico estruturante da composição da coalizão, enquanto a coordenação coletiva permanece como elemento associado e condicionado ao primeiro (Leifeld, 2013).

A noção de sistema de crenças do MCD se baseia em uma estrutura hierárquica, formada por três níveis (Quadro 1).

\section{QUADRO 1 NÍVEIS DO SISTEMA DE CRENÇAS DE COALIZÕES DE DEFESA}

\begin{tabular}{|c|c|}
\hline Níveis de crença & Descrição \\
\hline $\begin{array}{l}\text { Crenças essenciais } \\
\text { profundas }\end{array}$ & $\begin{array}{l}\text { Crenças mais abrangentes e estáveis referentes a valores normativos e ontológicos gerais aplicáveis } \\
\text { a múltiplos subsistemas. Exemplos incluem crenças relacionadas a projetos políticos (crenças } \\
\text { progressistas ou conservadoras). }\end{array}$ \\
\hline $\begin{array}{l}\text { Crenças essenciais da } \\
\text { política }\end{array}$ & $\begin{array}{l}\text { Orientações normativas e empíricas condizentes ao subsistema específico de uma política. As } \\
\text { concepções normativas se relacionam às diretrizes básicas e valores prioritários e as concepções } \\
\text { empíricas se associam às análises sobre problemas da política, proposição de causas e alternativas } \\
\text { de soluções. Estas crenças estão em um nível intermediário, embora sejam de difícil mudança, são } \\
\text { mais prováveis de serem ajustadas do que as crenças profundas. }\end{array}$ \\
\hline $\begin{array}{l}\text { Aspectos secundários } \\
\text { ou auxiliares }\end{array}$ & $\begin{array}{l}\text { Diretrizes sobre um subconjunto temático ou instrumentos específicos de tomada de decisão, } \\
\text { implementação ou controle no subsistema da política. Apresentam um escopo mais restrito, } \\
\text { predominantemente baseados em vivências empíricas, e mais propenso a aprendizados e mudanças. }\end{array}$ \\
\hline
\end{tabular}

Fonte: Adaptado de Weible, Sabatier, e McQueen (2009). 
As hipóteses de diferenciação e relação hierárquica no sistema de crenças e seus efeitos sobre a coordenação de atividades em coalizões são mantidas em revisões mais recentes sobre o MCD (Jenkins-Smith et al., 2017) e sobre o conceito de coalizão de defesa (Weible et al., 2019). Mas, algumas pesquisas aplicadas têm demonstrado dificuldade metodológica em distinguir os níveis, bem como, eventualmente, não encontraram sustentação empírica à tal estrutura hierárquica de crenças. Por isso, os autores observam que há possibilidade de reunir crenças essenciais da política e aspectos secundários, dependendo do escopo focal da pesquisa. Esta perspectiva integrada (não-hierárquica) foi a adotada na presente pesquisa.

Apesar da prevalência da análise do sistema de crenças em pesquisas aplicadas do MCD, Weible et al. (2019) ressaltam também a importância da análise sistemática das condições e atributos de coordenação dos atores para compreensão do modus operandi das coalizões.

O tipo ideal de coalizão de defesa, proposto por Weible et al. (2019), contempla a existência de atores no processo da política que (a) compartilham crenças, (b) coordenam suas ações e (c) exercem essas características sistematicamente ao longo do tempo. A partir deste tipo ideal, a ausência de atributos é analisada pelos autores enquanto formas alternativas de composição de coalizões de defesa, tais como formas efêmeras (cuja coordenação coletiva não se torna regular ao longo do tempo) ou formas latentes ou desconectadas (na ausência de coordenação).

Para refinar a análise de coalizões de defesa, Weible et al. (2019) propuseram cinco (5) atributos: atores da política, sistema de crenças, coordenação de atividades políticas, recursos e estabilidade. Entendemos que esta análise de atributos em coalizões de defesa contribui com um dos pontos anteriormente realçados na agenda de pesquisa do MCD, por Jenkins-Smith et al. (2014), em termos de examinar como uma coalizão de defesa se forma e como desenvolve sua estabilidade ao longo do tempo.

\subsection{Coalizão de Defesa e Atores Chaves no Processo de Mudança da Política}

Entender processos de mudanças no longo prazo em políticas públicas foi um dos focos analíticos originais na proposição do MCD. Para o modelo, as coalizões de defesa são quem geralmente assumem o protagonismo de tais mudanças ou de manutenção da estabilidade em um subsistema de política (Sabatier \& Jenkins-Smith, 1993).

Jenkins-Smith et al. (2017) mantêm quatro proposições sobre trajetórias de mudanças no MCD:

(1) as advindas de alterações ou perturbações externas ao subsistema, nas categorias de parâmetros relativamente estáveis ou de eventos dinâmicos;

(2) as baseadas em eventos internos e ações dos atores no subsistema;

(3) as geradas por meio de processos de aprendizados orientados à política; e

(4) as promovidas por acordos negociados entre coalizões previamente em forte oposição, em geral, facilitadas por organizações intermediárias.

Considera-se necessária a ocorrência de, ao menos, uma dessas trajetórias à mudança da política. Contudo, a ocorrência por si só de uma delas não é comumente suficiente à mudança, sendo requerida uma combinação destas na efetivação de processos de mudança (Jenkins-Smith et al., 2017). 
Entre as fontes de mudanças estão os processos de aprendizado orientados para a política, outro foco analítico da origem do MCD. Jenkins-Smith et al. (2014) associam os processos de aprendizado ao reforço, ou à necessidade de revisão, de preceitos do sistema de crenças de atores em uma coalizão. Estes processos de aprendizados têm implicações tanto sobre a concepção de problemas e soluções que devem ser direcionados no subsistema da política, quanto sobre as estratégias e instrumentos a serem utilizados para alcance de objetivos.

Jenkins-Smith et al. (2017) elencam quatro (4) fatores que influenciam os processos de aprendizados dos atores da política no MCD: (1) nível de conflito no subsistema; (2) estímulos existentes para o aprendizado; (3) atributos dos atores (crenças extremas ou moderadas, recursos de acesso a informações, estratégias) e (4) atributos dos fóruns e arenas de interação. Neste último, ressalta-se uma das hipóteses do modelo em que o aprendizado orientado para política é mais provável de se desenvolver quando existe um fórum de debates profissional no subsistema, o qual seja (a) prestigiado o suficiente para forçar membros de coalizões diferentes a participarem e (b) regido por normas profissionais (Weible et al., 2009).

Embora o aprendizado orientado para a política nas hipóteses originais do MCD esteja mais direcionado para a análise do processo da política em si, consideramos que possa ter relevância para a formação e desenvolvimento de coalizões de defesa, especificamente. Isso porque os processos de aprendizado podem influenciar a construção de consensos e disseminação de crenças e valores de uma coalizão em formação no subsistema, bem como incidir sobre a criação de mecanismos para mobilização e coordenação coletiva interna.

Béland e Cox (2015) ressaltaram o papel da disseminação de ideias e da conformação de interesses de diferentes grupos para a formação de coalizões. Estes autores apontaram que estas ideias tendem a ser polissêmicas no processo de formação de coalizões e que o empreendedor da política assume o papel de responsável em promover tais ideias e mobilizar apoiadores e, portanto, em formar coalizões.

A categoria de empreendedor da política é mais evidente em outros modelos analíticos, especialmente em destaque no modelo de múltiplos fluxos (Multiple Streams Framework - MSF) (Kingdon, 2014). No MCD, esta categoria não havia sido originalmente desenvolvida, mas Mintrom e Vergari (1996) já haviam postulado a possibilidade de sua incorporação para refinamento do modelo. Para Mintrom e Vergari (1996), o conceito de empreendedor da política iluminaria particularmente a análise da formação de coalizões de defesa (composição e coordenação de membros) e para alcançar maior detalhamento de como e quando ocorrerem mudanças críticas.

Posteriormente, Mintrom e Norman (2009) avançam na definição de papéis de empreendedores de política ao destacar quatro elementos chaves de atuação destes: demonstrar acuidade social, definir problemas, formar equipes e liderar por exemplo. Esses autores acreditam que a integração do conceito de empreendedor de política em modelos de análise de política pode proporcionar visões mais abrangentes dos múltiplos fatores envolvidos nos processos de mudança.

Capella (2016) buscou explorar o papel do empreendedor da política em múltiplos modelos analíticos, entre esses incluiu o MCD. Embora o MCD não explicite o papel de empreendedor da política, a autora realçou as relações do empreendedor com funções relacionadas à formação de coalizões e processos de aprendizados baseados em ideias. Ela destacou duas dimensões básicas da atuação dos empreendedores: a defesa de ideias no processo da política e a condução de processos de mudanças rápidas em políticas. 
Weible et al. (2019) citam os policy brokers e os empreendedores da política entre os diferentes atores no processo de política e possíveis membros de coalizões de defesa. Originalmente, o MCD já ressaltava a categoria de policy brokers. Policy brokers são atores geralmente investidos em cargos eletivos ou servidores públicos de alto escalão na política, cuja preocupação principal está em manter o nível de conflitos políticos no subsistema em limites aceitáveis e, quando necessário, buscam negociar soluções razoáveis entre coalizões oponentes (Sabatier \& Jenkins-Smith, 1993). Contudo, Sabatier e Jenkins-Smith (1993) ressalvam que, independente da atuação como policy brokers, esses indivíduos também poderiam ser membros de coalizão de defesa ou expressar posições mais favoráveis a determinada coalizão.

Neste artigo, buscamos focalizar as ações destes atores especificamente no processo de formação de coalizão. Pretendemos, de forma exploratória, observar e caracterizar os papeis exercidos, em termos de defesas de ideias, constituição de crenças e condução de processos de aprendizados e disseminação, construção de mecanismos de coordenação de atividades políticas, acumulação de recursos e estabilidade de atuação.

\section{METODOLOGIA}

Para analisar a trajetória dos processos de política de TA e TS no governo subnacional do estado do Pará (Brasil), utilizamos o método de pesquisa orientada por caso (Ragin, 1992, 2009), com abordagem qualitativa. O processo metodológico de casing foi usado para operacionalizar a categoria de subsistema da política, principal unidade de análise do MCD. Conforme Ragin (1992, 2009), casing se refere ao passo metodológico de constituir (delimitar e declarar) casos de pesquisa, por meio do "encaixe" processual de categorias teóricas-conceituais com as evidências empíricas e especificidades do fenômeno.

Para delimitar os casos dos processos de política de TA e TS de acordo com o conceito de subsistema de política, realizamos o casing em duas etapas: a primeira envolveu obter uma visão geral da política estadual de CT\&I no Pará; e a segunda correspondeu ao recorte específico dos processos de política vinculados às temáticas de TA e TS.

Na primeira etapa, utilizamos a noção convencional de unidade federativa do estado do Pará e as organizações de política nela situadas como diretrizes de delimitação geográfica. O foco esteve na identificação das organizações e atores envolvidos no processo de política associado à CT\&I. As três principais organizações identificadas foram a secretaria estadual responsável pelo domínio de CT\&I, a Fundação de Amparo à Pesquisa (FAP) estadual e a Universidade do Estado do Pará (UEPA).

Nesta primeira etapa, a coleta de dados se baseou em pesquisa documental dos últimos três planos plurianuais (PPA) do Pará (no período de 2008 a 2017), com foco aos programas relacionados à pasta de CT\&I (busca temática) e aos programas em que as três organizações chaves participavam (busca por organizações governamentais). Também realizamos análise documental nos planos diretores de CT\&I, diretrizes e instrumentos de financiamentos (editais e chamadas públicas) no mesmo período. Além disso, fizemos visitas e entrevistas exploratórias com servidores públicos das três organizações principais apontadas. A partir destas análises, verificamos as temáticas de inclusão social que alcançaram agenda e implementação no processo de política e seus atores principais.

A segunda etapa de delimitação dos dois subsistemas de política (TA e TS) abrangeu o mapeamento dos principais atores envolvidos no processo da política destas temáticas. A partir deste, examinamos a 
trajetória do processo de política, identificamos atores da política em papéis chaves e possíveis formação de coalizões de defesa locais. O período de análise dessas temáticas contemplou da sua ascensão à agenda estadual de CT\&I (ambas em 2011), buscando considerar seus antecedentes, até 2018.

A coleta de dados nesta segunda fase envolveu nova pesquisa documental e entrevistas semiestruturadas. A pesquisa documental compreendeu o financiamento de projetos e atividades relacionados a TA e TS realizadas por meio da Secretaria Estadual responsável pela pasta de CT\&I e pela FAP. As documentações pesquisadas foram os editais de financiamento, os respectivos projetos financiados e seus relatórios técnicos, além dos processos administrativos vinculados a estes e eventos temáticos realizados no estado.

Posteriormente à pesquisa documental, foram realizadas entrevistas semiestruturadas com os dirigentes da Secretaria Estadual e FAP, servidores públicos destas organizações envolvidos em processos de formulação dos editais e avaliação dos projetos, coordenadores dos projetos financiados e três pesquisadores relatados como influentes localmente nos temas durante as primeiras entrevistas. Assim, realizou-se entrevistas com 18 atores relacionados à TA e 13 atores relacionados à TS, de setembro de 2018 a janeiro de 2019.

A partir da análise documental e das entrevistas foram organizadas linhas do tempo com os marcos dos processos de cada política (TA e TS), as quais serviram de base para uma visão longitudinal dos casos, relevante para análise de longo prazo do MDC e reconstrução descritiva de cada trajetória (apresentadas nas seções seguintes).

Além disso, também foram realizadas as análises sobre o sistema de crenças dos atores de cada subsistema (TA e TS). As crenças dos atores foram analisadas usando o método de análise de discurso. Embora o método originalmente mais corrente no MDC fosse a análise de conteúdo, Jenkins-Smith et al. (2014) ressaltaram a abertura para uma orientação metodológica mais ampla com abordagens sobre representação de mundo ou aspectos ideológicos na política.

Neste estudo, a análise de discurso em cada processo de política tecnológica (TA e TS) buscou identificar as preferências e posições discursivas dos atores quanto a 6 categorias estabelecidas: (1) Enfoque principal do desenvolvimento tecnológico, (2) concepção de tecnologia, (3) quem produz conhecimento e tecnologia na sociedade, (4) quem são dos protagonistas da política em foco, (5) estratégias de implementação, e (6) estratégias de disseminação tecnológica.

\section{RESULTADOS E ANÁLISES}

\subsection{A Política Estadual de CT\&I no Pará e o Fomento à Tecnologia Assistiva e Tecnologia Social}

A partir da análise das fontes documentais da política estadual de CT\&I no Pará, no período de 2008 a 2017, constatamos um processo incremental em torno da concepção de sistema de inovação (Sistema Paraense de Inovação). Esta concepção realça o papel da política de inovação tecnológica para geração de desenvolvimento para o estado, com ênfase em sua dimensão econômica, conforme exemplos de trechos documentais abaixo citados.

Fortalecer a economia a partir da atração de plantas industriais e do desenvolvimento de produtos inovadores em ambientes como: distritos e áreas industriais, parques de ciência e tecnologia e incubadoras de empresas (Pará, 2007, p. 90). 
Composto por organizações, pessoas, instrumentos e processos, o SPI (Sistema Paraense de Inovação) visa ao engajamento do Estado do Pará na nova economia mundial - fortemente baseada no conhecimento - e se configura como alternativa de governança garantidora, em caráter duradouro e sustentável, do desenvolvimento regional/local de longo prazo, gerador de melhor qualidade de vida às futuras gerações (Secti, 2011, p. 63).

Tal constatação alerta à necessidade de o Sistema Paraense de Inovação ser fortalecido, organizado e, ao mesmo tempo, direcionado para processos produtivos mais baseados em conhecimento, em consonância com o potencial de geração de riquezas em âmbito regional (Sectet, 2015, p. 24).

Contudo, a maior regularidade e financiamento na política neste período se manteve associado às modalidades de financiamento relacionadas à concessão de bolsas e apoio à pós-graduação stricto sensu e à pesquisa científica e tecnológica. Observarmos que dos 124 editais identificados lançados pela FAP estadual de 2008 a 2017, 48 eram para pesquisa científica e tecnológica, 19 para bolsas em programas de pós-graduação, 11 para programas de iniciação científica e somente 6 para pesquisa e desenvolvimento (P\&D) e inovação em empresas.

Esta observação corrobora com outros estudos sobre a política de CT\&I brasileira (Dagnino, 2016; Dias, 2012), em que, apesar da ênfase na agenda em prol da inovação tecnológica, este destaque teve resultados restritos, mais voltados à legitimação do fomento público para C\&T. Isso em função de condições macroeconômicas e periféricas desfavoráveis (elevadas taxas de juros, economia baseada exportação de commodities, desarticulação da política industrial, dependência tecnológica e cultural) que têm implicado em uma baixa propensão para inovar pelo empresariado local. Consequentemente, a política de CT\&I no Brasil permanece sob o predomínio de atores vinculados a comunidade de pesquisa (Dagnino, 2016), mesmo com a ascensão à agenda da inovação tecnológica empresarial.

Especificamente quanto as temáticas de inclusão social no processo da política de CT\&I no Pará, encontramos nos principais planos de governo quatro temas: TS, inclusão digital, agricultura familiar e desigualdade territorial nos investimentos e capacidades de CT\&I no estado. No que tange a execução do fomento via editais, identificamos onze (11) temas associados a tais temáticas: TS, inclusão digital, agricultura familiar, desigualdade territorial, pessoas com deficiência e idosos, doenças negligenciadas, gênero, crianças e adolescentes, populações tradicionais, economia inclusiva e acesso equitativo a água.

Embora estes temas, em geral, não apresentassem centralidade na orientação da política ou volume de financiamento substancial, suas aparições são indicativos de inserção de novas pautas na agenda da política. Dentre esses temas, selecionamos dois para análise específica.

O tema de TS esteve presente em documentos norteadores da política de CT\&I a partir de 2011 e foi o único com financiamento via edital da Secretaria Estadual, onde havia uma unidade administrativa dedicada ao tema. Assim, entendemos que seus atores possam ter buscado uma estratégia de institucionalização junto às instâncias de decisão internas e mais tradicionais na política estadual de CT\&I.

O tema de TA não foi citado nos documentos norteadores da política, no entanto, foi objeto de dois editais exclusivos via FAP e linha temática de outros dois, o que pode indicar que os atores da política tenham buscado estratégias alternativas para alcance da agenda. 


\subsection{Subsistema da Política Estadual de Fomento à Tecnologia Assistiva (TA)}

Uma política de fomento à TA na agenda estadual de CT\&I no Pará começou a emergir em 2011 e se concretizou em 2013, com o lançamento do primeiro edital específico para desenvolvimento de TA pela FAP estadual (no 010/2013) no âmbito de um programa intersetorial.

Identificamos que o papel chave na ascensão da temática à agenda foi de uma pesquisadora que já tinha um histórico de atuação no atendimento a pessoas com deficiência. Em 1997, ela havia fundado a Associação de Assistência à Criança Deficiente da Amazônia (ACDA) e, desde meados da década de 2000, vem coordenando um núcleo de TA (Nedeta) na Universidade do Estado do Pará (UEPA), o qual vinha se estruturando com apoio de financiamentos de agências de fomento federais (Finep e CNPq).

Desde 2006, esta pesquisadora organizava fóruns bianuais sobre a temática de TA por meio do Nedeta. Em 2011, ela conseguiu captar recursos para organização da $1^{\text {a }}$ Feira Paraense de Tecnologia Assistiva, Acessibilidade e Inclusão Social das Pessoas com Deficiência que foi realizada em parceria com a Secretaria de estadual responsável pela pasta da assistência social. A escolha dessa parceria pela pesquisadora visava promover maior sensibilização e articulação com pessoas chaves no governo estadual, alcançando a visita do próprio governador do estado na feira.

Neste mesmo ano, o governo federal lançou o Plano Nacional dos Direitos das Pessoas com Deficiência (PNDPcD) (decreto $\mathrm{n}^{\circ} 7.612 / 2011$ ), que proporcionou visibilidade às políticas para assegurar direitos às pessoas com deficiência (incluso as TA) e, também, previa a indução de descentralização de ações aos governos estaduais e municipais. Esta convergência entre estímulos externos ao subsistema (PNDPcD) e de iniciativas locais de TA permitiu a formulação do Plano Existir (programa estadual articulado ao PNDPcD) e o compromisso do governador estadual neste plano de financiar o desenvolvimento de TA no domínio da CT\&I.

Por meio do edital $n^{\circ}$ 010/2013 da FAP estadual foram financiados 15 projetos de TA ( 5 associados ao Nedeta). Em 2016, foi realizada a I Feira Estadual Existir, II Feira Paraense de Tecnologia Assistiva, Acessibilidade e Inclusão Social da Pessoa com Deficiência e o V Fórum de Tecnologia Assistiva e Inclusão Social da Pessoa com Deficiência. E, em 2017, lançado o segundo edital exclusivo ao fomento de TA ( $\left.{ }^{\circ} 012 / 2017\right)$.

Consideramos que a pesquisadora em questão se caracteriza enquanto uma empreendedora da política de TA, seja na defesa de ideias, seja na atuação em múltiplas frentes para mudanças no processo de política e alcance da agenda (Capella, 2016; Kingdon, 2014).

Dada a marginalidade da TA na agenda de CT\&I, a empreendedora buscou apoio junto ao subsistema da política de assistência social e ao chefe do executivo estadual (governador do estado). Assim, ela articulou encadeamentos de outros subsistemas para influenciar o processo da política estadual de CT\&I e teve senso de oportunidade para aproveitar a ascensão do tema na agenda da política federal.

Além da articulação de ações para o fomento à TA, houve a manutenção da organização de espaços de interação, compartilhamento de crenças e aprendizados (fóruns, feiras e elaboração conjunta de livros) no subsistema específico em desenvolvimento, cujo papel chave também é creditado a mesma empreendedora da política mencionada.

Antes do tema de desenvolvimento de TA alcançar à agenda estadual, não observávamos a existência prévia de uma coalizão de defesa ao tema. A chamada para financiamento de projetos 
ampliou o número de atores locais envolvidos com a temática. A organização de ações de divulgação e fóruns (acadêmicos e da política) têm conduzido a aprendizados e à agregação de parte considerável destes pesquisadores em torno do Nedeta. Portanto, entendemos que a trajetória do subsistema de política de fomento à TA tem propiciado estímulos à formação e fortalecimento de uma coalizão de defesa em prol de TA no estado.

Em termos do sistema de crenças relacionado ao desenvolvimento de TA, observamos que os pesquisadores tendem a se associar ao chamado modelo social de deficiência (Garcia, 2014). Mas, notamos um desdobramento deste modelo em duas direções com ênfases distintas, uma para mecanismos de mercado (denominamos de ênfase tecnológica-mercadológica) e outra para mecanismos mais plurais socialmente (denominamos de ênfase social), conforme quadro 2.

\section{QUADRO 2 SÍNTESE DOS PRINCIPAIS PONTOS DE CONCEPÇÃO DE TA DE ÊNFASE TECNOLÓGICA- MERCADOLÓGICA E DE ÊNFASE SOCIAL}

\begin{tabular}{|c|c|c|}
\hline $\begin{array}{l}\text { Elementos de } \\
\text { crenças }\end{array}$ & Concepção de ênfase tecnológica-mercadológica1 & Concepção de ênfase social \\
\hline Enfoque principal & $\begin{array}{l}\text { Acesso inadequado da população que precisa } \\
\text { de TA por um desencontro entre objetivos e } \\
\text { capacidades destas pessoas e os recursos } \\
\text { existentes no ambiente e a "medicalização", que } \\
\text { leva a segregação e altos níveis de pobreza nesta } \\
\text { população }\end{array}$ & $\begin{array}{l}\text { Acesso restrito da população que necessita de TA, } \\
\text { por um desencontro entre uma restrita demanda } \\
\text { relativa (capacidade de compra) e um amplo } \\
\text { conjunto de necessidades não atendidas e não } \\
\text { atendíveis via condições de mercado, devido a altos } \\
\text { níveis de pobreza nesta população }\end{array}$ \\
\hline $\begin{array}{l}\text { Concepção de } \\
\text { tecnologia }\end{array}$ & $\begin{array}{l}\text { Desenvolvimento de tecnologia direcionado às } \\
\text { expectativas e necessidades do consumidor; por } \\
\text { um sistema guiado pelo mercado }\end{array}$ & $\begin{array}{l}\text { Tecnologia desenvolvida em interação com usuário } \\
\text { com orientações voltadas para critérios de baixo } \\
\text { custo ou economicamente acessíveis }\end{array}$ \\
\hline $\begin{array}{l}\text { Quem produz } \\
\text { conhecimento e } \\
\text { tecnologia }\end{array}$ & $\begin{array}{l}\text { Cientistas, engenheiros e pessoas com } \\
\text { deficiência, enquanto consumidores }\end{array}$ & $\begin{array}{l}\text { Cientistas, engenheiros e pessoas com deficiência, } \\
\text { enquanto cidadãos }\end{array}$ \\
\hline $\begin{array}{l}\text { Protagonistas da } \\
\text { política }\end{array}$ & Cientistas, governo e empresas & $\begin{array}{l}\text { Cientistas, governo, empresas, pessoas com } \\
\text { deficiência e organizações representativas }\end{array}$ \\
\hline $\begin{array}{l}\text { Estratégia de } \\
\text { implementação }\end{array}$ & $\begin{array}{l}\text { A remoção de barreiras sistêmicas e estruturais, } \\
\text { pela internalização de um "status" de consumidor } \\
\text { e externalização da ação de adquirir recursos }\end{array}$ & $\begin{array}{l}\text { Engajamento social e político da pessoa com } \\
\text { deficiência, pela internalização de um "status" } \\
\text { de cidadão e externalização da ação de buscar } \\
\text { equiparação de direitos }\end{array}$ \\
\hline $\begin{array}{l}\text { Disseminação } \\
\text { tecnológica }\end{array}$ & Oferta de produtos e serviços via mercado & $\begin{array}{l}\text { Múltiplos canais de disseminação, inclusive públicos } \\
\text { e cooperativos }\end{array}$ \\
\hline
\end{tabular}

${ }^{1}$ Elementos parcialmente inspirados no programa de certificação em aplicações da tecnologia assistiva da California State University Northridge citado em: BRASIL. Subsecretaria Nacional de Promoção dos Direitos da Pessoa com Deficiência. Comitê de Ajudas Técnicas. Tecnologia Assistiva. Brasília: CORDE, 2009.

Fonte: Elaborado pelos autores. 
A maioria dos projetos financiados pelo edital de 2013 desenvolveu aplicações de TA com preocupação de serem economicamente viáveis e passíveis de serem disseminadas via políticas públicas ou por canais gratuitos, embora tais condições não fossem exigidas no edital. Observamos ainda que dos 15 projetos, 9 alcançaram o desenvolvimento de produtos ou serviços de TA efetivamente aplicados junto ao público-alvo (condição também não exigida no edital). Em todos esses projetos com atendimentos efetivados foram usados canais de disseminação via serviços públicos (com ações intersetoriais nas políticas de educação e saúde) ou gratuitos. Nenhum projeto foi desenvolvido em parceria com empresa ou teve iniciativas posteriores de transferência das soluções tecnológicas para empresas. Isso tenderia a caracterizá-los enquanto predominantemente de concepção socialmente orientados.

No entanto, a participação das pessoas com deficiência nestes projetos foi principalmente em um papel de beneficiárias ou usuárias dos produtos e serviços. Não identificamos projetos em que pessoas com deficiência ou suas organizações representativas tenham feito parte das equipes de formulação ou P\&D. Também não houve relato que estas tenham participado de instâncias de formulação ou avaliação do edital junto à FAP. As equipes de projetos foram em sua maioria formadas somente por pesquisadores e o processo de avaliação previsto baseado na análise por pares dos relatórios de pesquisa.

Estas características restringem processos de inclusão social por meio do engajamento social e político das pessoas com deficiência (MacLachlan et al., 2018). Diante disso, cabe concordamos com Garcia (2014), que ainda há tensões relevantes entre práticas valorizadas pelo modelo social de deficiência e as práticas institucionalizadas de pesquisa e política de CT\&I no país. Visto que estas últimas permanecem majoritariamente centradas nos pesquisadores, com barreiras a participação de outros atores, inclusive seus públicos-alvo, neste caso pessoas com deficiência.

Compreendemos que a temática de TA é uma área de pesquisa em desenvolvimento inicial nas organizações do complexo de C\&T estadual. Muitos pesquisadores entrevistados ingressaram na área a partir do edital de 2013, estando em processo de aprendizado. Conforme entrevistas com estes pesquisadores não foi frequente expressões de conflitos de crenças na política. Estes tendiam a posições menos divergentes das práticas de $\mathrm{P} \& \mathrm{D}$ predominantemente estabelecidas, principalmente pelo não tensionamento sobre a participação limitada de pessoas com deficiência nos projetos e no próprio âmbito da política de CT\&I.

Em síntese, a política estadual de fomento à TA no Pará emerge por um encadeamento de eventos externos (como o PNDPcD e o compromisso do executivo central do estado) mobilizados por atores internos, especialmente por uma empreendedora da política. A partir do MCD, dois papéis da empreendedora da política são realçados: (1) promoção e manutenção de fóruns para compartilhamento de crenças e processos de aprendizados (Weible et al., 2009); e (2) coordenação de ações coletivas tanto relacionadas a estes fóruns, quanto na implementação de projetos de desenvolvimento de TA associados ao seu Núcleo. Estes aspectos aproximam-se dos elementos destacados por Mintrom e Norman (2009), particularmente, a formação de equipes, no caso da coalizão de defesa.

A análise de maior prazo associada ao MCD, quando aplicado à visão da trajetória da empreendedora, também demonstra os recursos da política acumulados por esta ao longo do tempo (seja de recursos materiais como financiamentos, seja de recursos sociais como reputação) para articulá-los no momento adequado de ascensão à agenda. Bem como, permite observar uma 
continuidade de atuação em todo o processo da política (não somente na definição da agenda), como na implementação de programas e projetos.

\subsection{Subsistema da Política Estadual de Fomento à Tecnologia Social (TS)}

O processo da política de fomento à TS emergiu na agenda estadual de CT\&I no Pará em 2011 com sua inclusão no programa de CT\&I do PPA estadual e no Plano Diretor de CT\&I. Em 2012, uma Diretoria de Tecnologia Social (DTS) foi implementada na estrutura da Secretaria estadual de CT\&I. Papel chave nesta ascensão foi creditado ao dirigente da Secretaria que propôs a temática nos instrumentos da política e apoiou a estruturação da Diretoria.

A partir deste início, identificamos dois estágios distintos na trajetória do processo de política de TS no governo estadual. O primeiro, de 2011 a 2014, desenvolveu-se a partir da estruturação da DTS e teve como ênfase a promoção da política de TS por meio da institucionalização de uma Rede de TS no Pará (RTS-PA), aos moldes da Rede de TS nacional (RTS Brasil). Neste sentido, foram realizados Fóruns de TS estaduais e elaborado um documento de referência para a RTS-PA, com a proposição de uma estrutura de governança própria à política estadual de TS.

Neste estágio, percebemos forte preocupação com a conceituação de TS e seu alinhamento à concepção pactuada na RTS Brasil. Interpretamos que este alinhamento correspondia à filiação de crenças dos principais atores locais envolvidos. Ao mesmo tempo em que tal associação à concepção nacional também representava uma estratégia de legitimação da temática perante demais atores da comunidade de pesquisa e dirigentes da política estadual de CT\&I.

Embora os atores locais não relatem indução do governo federal à ascensão da temática de TS à agenda estadual, eles admitem ter buscado mobilizar processos de aprendizados em relação à estrutura e governança da RTS nacional para organizar a RTS-PA e criar fóruns de TS estaduais. Além disso, grupos de pesquisas e organizações da sociedade civil (OSC) envolvidas em projetos de TS financiados pelo governo federal foram convidados a participar destes fóruns.

Contudo, cabe destacar que as crenças relacionadas à concepção de TS dos principais atores envolvidos nestas ações apresentava divergência às crenças predominantes da política de CT\&I, representadas no direcionamento principal da política de inovação tecnológica, conforme sintetizamos no quadro 3.

\section{QUADRO 3 SÍNTESE DOS PRINCIPAIS PONTOS DE CONCEPÇÃO PREDOMINANTE DA POLÍTICA ESTADUAL DE CT\&I E DA POLÍTICA DE TS NO 1 ESTÁGIO DA TRAJETÓRIA ESTUDADA}

\begin{tabular}{|c|c|c|}
\hline $\begin{array}{l}\text { Elementos de } \\
\text { crenças }\end{array}$ & $\begin{array}{l}\text { Concepção predominante na política estadual de } \\
\qquad \text { CT\&l }\end{array}$ & $\begin{array}{l}\text { Concepção da política de tecnologia social } \\
\qquad\left(1^{\circ} \text { estágio }\right)\end{array}$ \\
\hline Enfoque principal & $\begin{array}{l}\text { Inovação tecnológica gera desenvolvimento } \\
\text { econômico (via competitividade e agregação } \\
\text { de valor) que transborda benefícios sociais por } \\
\text { melhoria de empregos, qualificação profissional e } \\
\text { novos produtos }\end{array}$ & $\begin{array}{l}\text { Processos de exclusão sociais são estruturais. } \\
\text { A inclusão social e produtiva perpassa por } \\
\text { desenvolvimentos tecnológicos alternativos, } \\
\text { adequados à realidade e grupos sociais locais }\end{array}$ \\
\hline
\end{tabular}




\begin{tabular}{|c|c|c|}
\hline $\begin{array}{l}\text { Elementos de } \\
\text { crenças }\end{array}$ & $\begin{array}{l}\text { Concepção predominante na política estadual de } \\
\text { CT\&I }\end{array}$ & $\begin{array}{l}\text { Concepção da política de tecnologia social } \\
\qquad\left(1^{\circ} \text { estágio }\right)\end{array}$ \\
\hline $\begin{array}{l}\text { Concepção de } \\
\text { tecnologia }\end{array}$ & $\begin{array}{l}\text { Tecnologia desenvolvida predominantemente } \\
\text { baseada em conhecimentos científicos avançados }\end{array}$ & $\begin{array}{l}\text { Tecnologia desenvolvida pela interação com } \\
\text { a comunidade ou grupo social, baseada em } \\
\text { diferentes tipos de conhecimentos, incluindo } \\
\text { populares e tradicionais }\end{array}$ \\
\hline $\begin{array}{l}\text { Quem produz } \\
\text { conhecimento, } \\
\text { tecnologia e } \\
\text { inovação }\end{array}$ & $\begin{array}{l}\text { Cientistas, engenheiros e empreendedores/ } \\
\text { empresários }\end{array}$ & $\begin{array}{l}\text { Cientistas e diferentes atores sociais por meio de } \\
\text { redes de saberes }\end{array}$ \\
\hline $\begin{array}{l}\text { Protagonistas da } \\
\text { política }\end{array}$ & Cientistas, governo e empresas & $\begin{array}{l}\text { Cientistas, governo, empresas, OSC e } \\
\text { comunidades ou grupos sociais locais }\end{array}$ \\
\hline $\begin{array}{l}\text { Estratégia de } \\
\text { implementação }\end{array}$ & $\begin{array}{l}\text { Ênfase em organizações tradicionais do setor } \\
\text { produtivo, empresas e aglomerados industriais }\end{array}$ & Ênfase ao cooperativismo ou associativismo \\
\hline $\begin{array}{l}\text { Disseminação } \\
\text { tecnológica }\end{array}$ & Transferência de tecnologia & Reaplicação e adequação sociotécnica \\
\hline
\end{tabular}

Fonte: Elaborado pelos autores.

Essa divergência de crenças tensionava a temática de TS a uma posição marginal, conceitual e materialmente, no âmbito da política de CT\&I. Assim, apesar da presença na política explícita neste período (2011 a 2014), não houve relevante financiamento estadual direto a projetos de TS. Somente dois (2) financiamentos de baixo volume a projetos de empreendimentos comunitários foram identificados em 2012.

O segundo estágio na trajetória da política de TS foi caracterizado pela construção de um novo desenho institucional, no período de 2015 a 2017. Em 2015, a Secretaria Estadual deixa de ser exclusivamente dedicada a CT\&I, o que resultou em restrições orçamentárias à pasta e maior tensão por recursos internos. A pauta de TS foi mantida na agenda, mas as estruturas concebidas anteriormente (DTS, Fóruns e RTS-PA) foram preteridas. A temática ficou alocada no nível de Coordenadoria da Secretaria (Coordenadoria de Tecnologia para Desenvolvimento Social) subordinada a Diretoria de C\&T, com atrito entre as crenças predominantes da Diretoria de C\&T e dos atores até então envolvidos com TS.

O instrumento principal de implementação do fomento da política de TS passou a ser o financiamento direto de projetos de TS baseados no formato convencional de pesquisas de C\&T, coordenados por pesquisadores vinculados a universidades. Quatro (4) projetos foram financiados no período, com o processo decisório centrado na estrutura da Secretaria.

Entre os quatro projetos financiados, identificamos que atores da equipe de execução de três (3) deles apresentavam uma concepção de TS similar ou com relatos de aprendizados vinculados a segunda coluna do quadro 3, principalmente quanto a importância do desenvolvimento tecnológico ocorrer em interação com comunidades locais. Outro projeto, apesar de ser voltado ao atendimento de populações carentes, adotava uma concepção tecnológica alinhada àquela predominante na política geral de CT\&I (primeira coluna do quadro 3).

Apesar de verificarmos atores com crenças similares atuando em projetos financiados no subsistema estadual, estes relataram não haver interações entre si. Consequentemente, não 
identificamos coordenação de ação coletiva entre eles que pudesse caracterizar uma coalizão de defesa em desenvolvimento. Pela tipologia de Weible et al. (2019), a situação retrataria uma coalizão latente ou desconectada.

Em 2017, o Conselho Estadual da pasta de CT\&I aprovou uma resolução (CONSECTET $\mathrm{n}^{\circ} 003 / 2017$ ) dedicada à política estadual de TS, que instituiu um novo conjunto de diretrizes. Estas diretrizes apresentaram uma forte aproximação às concepções e instrumentos predominantes na política de CT\&I. A estrutura de governança e instrumentos da política de TS foram direcionados aos centros decisórios da Secretaria e suas organizações associadas (FAP e Parque de C\&T).

Esta institucionalização foi novamente creditada como iniciativa do mesmo dirigente da Secretaria. Entendemos que esta ação visou estabelecer um nível de compromisso razoável para o desenvolvimento da política de TS, por meio de sua legitimação perante as instâncias institucionais gerais da CT\&I estadual. Ao aproximar as diretrizes da política de TS àquelas predominantes na política de CT\&I geral, as divergências entre as concepções e instrumentos predominantes na política geral de CT\&I e concepções e instrumentos de desenvolvimento de TS tenderam a ser reduzidas. A atuação deste dirigente condiz com a descrição de policy broker de Sabatier e Jenkins-Smith (1993), enquanto mediador de disputas. Cabe indicar que as crenças da política deste dirigente eram filiadas à concepção predominante de CT\&I.

Apesar desta estratégia do policy broker ter contribuído, em certa medida, para a institucionalização da TS na agenda estadual, também permitiu uma abertura a maior variedade de crenças de atores no subsistema específico de TS, o que pode levar a uma maior concorrência por recursos neste. Inclusive, com a possibilidade de captura da temática de TS por atores com crenças mais próximas da coalizão de CT\&I predominante. Assim, houve uma redução do potencial conflitivo da temática de TS frente à política de CT\&I predominante, mas o subsistema de TS submete-se a um maior potencial de divergência interna em relação às crenças sobre TS.

Em síntese, um policy broker (dirigente na estrutura da política) favoreceu a ascensão da temática de TS à agenda e a formação de um subsistema específico de TS. Contudo, este policy broker não teve atuação direta sobre mecanismos de formação de coalizão de defesa (estabelecimento de mecanismos de defesa e compartilhamento de crenças ou coordenação de ações coletivas).

No primeiro estágio da trajetória da política, houve incentivos à estruturação de um corpo burocrático dedicado à política (DTS) e articulações para o compartilhamento de crenças e de aprendizados sobre TS (RTS-PA e fóruns). Estas iniciativas poderiam, em maior prazo, potencializar a formação de uma coalizão de defesa e o surgimento de empreendedores vinculados a esta, porém elas não foram perenes o suficiente para tal.

No segundo estágio, o policy broker foi bem-sucedido em institucionalizar a política de TS junto a instância superior da CT\&I e reduzir as divergências de concepções e instrumentos desta com a política predominante de CT\&I. Porém, não se observou nenhuma coalizão local de TS em desenvolvimento. Em parte, isso pode ser relacionado à ausência de fóruns, ao aumento de concorrência interna por recursos entre os atores no subsistema e à ausência de atores com crenças similares da política de TS que se dedicassem a atuar como empreendedores da política, incluindo na formação e desenvolvimento de uma coalizão de defesa. 


\section{CONSIDERAÇÕES FINAIS}

Esse artigo discutiu a formação de coalizões de defesa e os papéis de atores chaves, tendo como base as políticas de fomento à TA e TS no âmbito da CT\&I em contexto subnacional. Os dois processos de política analisados correspondem a pautas marginais de inclusão social na agenda CT\&I, mas apresentaram trajetórias e papéis de atores chaves distintos.

Em ambos os casos analisados, os atores chaves tiveram papel relevante no surgimento dos subsistemas de política (TA e TS), mas tiveram interações distintas quanto à formação de coalizão de defesa. No caso de TA, a atuação da empreendedora da política corroborou ser necessário para alcance e manutenção do tema na agenda e foi positivamente associada à formação de coalizão de defesa. Essa associação se relaciona a atuação em papéis de defesa da temática na agenda, de promoção de mecanismos regulares de compartilhamento de crenças e aprendizados (organização de eventos e coletânea de livros), de coordenação de ações coletivas (proposição de projetos e demandas de novos financiamentos) e de alcance de uma posição de reconhecimento científico e na política desta temática ao longo do tempo (recursos e estabilidade).

No caso da política de TS, não observamos uma coalizão de defesa em desenvolvimento, sendo ausente um ator com características associadas aos papéis de articulação sistemática para formação de coalizão de defesa na política.

Os casos analisados mostram que a incorporação da categoria de empreendedor de política no MCD ilumina detalhes de como e quando as mudanças no processo de política ocorrem e de sua influência na formação de coalizão de defesa. A análise do empreendedor de política, a partir da perspectiva analítica do MCD, conduz a uma visão de longo prazo sobre a atuação deste. Assim, demonstra-se como recursos e aprendizados são acumulados pelo empreendedor, como ele alcança estabilização e constrói estratégias. Também, implica em uma concepção do empreendedor de política fortemente associada à conceituação de coalizões de defesa (devido ao protagonismo destas na política no MCD), o que realça características de agrupamento de aliados por compartilhamento de crenças, de criação de fóruns e mecanismos de aprendizados orientados para a política e de atuação sistemática na coordenação de ações coletivas no subsistema. 


\section{REFERÊNCIAS}

Béland, D., \& Cox, R. H. (2016). Ideas as coalition magnets: coalition building, policy entrepreneurs, and power relations. Journal of European Public Policy, 23(3), 428-445. Recuperado de https://doi. org/10.1080/13501763.2015.1115533

Capella, A. C. N. (2016). Um estudo sobre o conceito de empreendedor de políticas públicas: ideias, interesses e mudanças. Cadernos EBAPE. $B R, 14$ (spe), 486-505. Recuperado de http://dx.doi. org/10.1590/1679-395117178

Capella, A. C. N., \& Brasil, F. G. (2015). Análise de políticas públicas: uma revisão da literatura sobre o papel dos subsistemas, comunidades e redes. Novos estudos CEBRAP, 101, 57-76. Recuperado de http:// dx.doi.org/10.1590/S0101-33002015000100003

Cavalcante, L. R. (2011). Desigualdades regionais em ciência, tecnologia e inovação (CT\&I) no Brasil: uma análise de sua evolução recente (Texto para Discussão, No. 1574). Brasília, DF: Instituto de Pesquisa Econômica Aplicada.

Coordenadoria Nacional para Integração da Pessoa Portadora de Deficiência. (2009). Tecnologia Assistiva. Brasília, DF: Subsecretaria Nacional de Promoção dos Direitos da Pessoa com Deficiência.

Dagnino, R. (2016). A Anomalia da Política de C\&T e sua Atipicidade Periférica. Revista Iberoamericana de Ciencia, Tecnología y Sociedad-CTS, 11(33), 33-63. Recuperado de http://dx.doi.org/10.1590/S010269092014000300004

Dias, R. de B. (2012). Sessenta anos de politica científica e tecnológica no Brasil. Campinas, SP: Editora Unicamp.

Faria, C. A. P. (2003). Ideas, knowledge and public policies: a brief inventory of the latest main analytical approaches. Revista Brasileira de Ciências Sociais, 18(51), 21-30. Recuperado de http://dx.doi. org/10.1590/S0102-69092003000100004

Garcia, J. C. D. (2014). Deficiência e Tecnologia Assistiva: Conceitos e Implicações para as Políticas Públicas. In Proceedings of $1^{\circ}$ International Assistive Technology Symposium (pp. 69-85), Campinas, SP.

Jenkins-Smith, H. C., Nohrstedt, D., Weible, C. M., \& Sabatier, P. A. (2014). The advocacy coalition framework: Foundations, evolution, and ongoing research. In P. Sabatier, \& C. Weible (Ed.), Theories

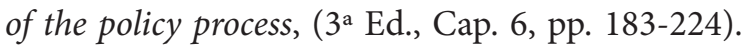
Boulder, CO: Westview Press.

Jenkins-Smith, H. C., Nohrstedt, D., Weible, C. M., \& Ingold, K. (2017). The advocacy coalition framework: An overview of the research program. In P. Sabatier, \& C. Weible (Ed.), Theories of the policy process (4 ${ }^{\text {a }}$ Ed., Cap. 5, pp. 135-171). New York, NY: Routledge.

Kingdon, J. W. (2014). Agendas, alternatives, and public policies. Harlow, Essex: Pearson Education Limited.

Law no. 7,077 (2020). Anexo Programa Finalístico. Belém, PA: Diário Oficial do Estado do Pará.

Leifeld, P. (2013). Reconceptualizing major policy change in the advocacy coalition framework: A discourse network analysis of German pension politics. Policy Studies Journal, 41(1), 169-198. Recuperado de https://doi.org/10.1111/psj.12007

MacLachlan, M., Banes, D., Bell, D., Borg, J., Donnelly, B., Fembek, M. ... Hooks, H. (2018). Assistive technology policy: a position paper from the first global research, innovation, and education on assistive technology (GREAT) summit. Disability and Rehabilitation: Assistive Technology, 13(5), 454466. Recuperado de https://doi.org/10.1080/174831 07.2018.1468496

Mintrom, M., \& Norman, P. (2009). Policy entrepreneurship and policy change. Policy Studies Journal, 37(4), 649-667. Recuperado de https://doi. org/10.1111/j.1541-0072.2009.00329.x

Mintrom, M., \& Vergari, S. (1996). Advocacy coalitions, policy entrepreneurs, and policy change. Policy studies journal, 24(3), 420-434. Recuperado de https://doi.org/10.1111/j.1541-0072.1996.tb01638.x

Ragin, C. C. (1992). "Casing" and the process of social inquiry. In C. C. Ragin \& H. S. Becker (Ed.), What is a case? Exploring the foundations of social inquiry (Cap. 10, pp. 217-226) New York, NY: Cambridge University Press.

Ragin, C. C. (2009). Reflections on casing and caseoriented research. In D. Byrne \& C. C. Ragin (ed.) The Sage handbook of case-based methods (cap. 31, pp. 522-534). London, UK: Sage Publications. 
Rede de Tecnologia Social (Org.). (2010). Tecnologia Social e Desenvolvimento Sustentável. Brasília/DF: Secretaria Executiva da RTS.

Sabatier, P. A., \& Jenkins-Smith, H. C. (1993). Policy change and learning: An advocacy coalition approach. Boulder, CO: Westview Press.

Secretaria de Estado de Ciência, Tecnologia e Educação Técnica e Tecnológica. (2015). Plano Diretor 2015-2019: Plano de CT\&I e Educação Profissional e Tecnológica. Belém, PA: SECTET. Recuperado de https://issuu.com/sectipa/docs/ plano_diretor_sectet_2015-2019/1

Secretaria de Estado de Ciência, Tecnologia e Inovação. (2011). Visão Estratégica: Plano Diretor de Ciência, Tecnologia 2011/2015. Belém, PA: SECTI,
2011. Recuperado de https://issuu.com/sectipa/ docs/planodiretorsecti

Theis, I. M., Strelow, D. R., \& Lasta, T. T. (2017). CT\&I e desenvolvimento desigual no Brasil: é possível outro "modelo de desenvolvimento"?. Revista Tecnologia e Sociedade, 13(27), 43-61. Recuperado de http://dx.doi.org/10.3895/rts.v13n27.3637

Weible, C. M., Sabatier, P. A., \& McQueen, K. (2009). Themes and variations: Taking stock of the advocacy coalition framework. Policy Studies Journal, 37(1), 121-140. Recuperado de https://doi.org/10.1111/ j.1541-0072.2008.00299.x

Weible, C. M., Ingold, K., Nohrstedt, D., Henry, A. D., \& Jenkins-Smith, H. C. (2019). Sharpening advocacy coalitions. Policy studies journal. Recuperado de https://doi.org/10.1111/psj.12360

\section{Diana Cruz Rodrigues}

https://orcid.org/0000-0001-6670-8907

Doutora em Administração; Professora na Universidade da Amazônia (UNAMA) no Programa de Pós-Graduação em Administração. E-mail: dicruzrodrigues@gmail.com

\section{Mário Vasconcellos Sobrinho}

https://orcid.org/0000-0001-6489-219X

Doutor em Estudos do Desenvolvimento; Professor na Universidade da Amazônia (UNAMA) no Programa de Pós-Graduação em Administração e na Universidade Federal do Pará (UFPA) no Programa de Pós-Graduação em Gestão de Recursos Naturais e Desenvolvimento Local. E-mail: mario.vasconcellos@unama.br

\section{Ana Maria de Albuquerque Vasconcellos}

https://orcid.org/0000-0002-7594-3578

Doutora em Estudos do Desenvolvimento; Professora na Universidade da Amazônia (UNAMA) no Programa de Pós-Graduação em Administração. E-mail: anamaria.vasconcellos@unama.br 